QCD Evolution Workshop 2013

International Journal of Modern Physics: Conference Series

Vol. 25 (2014) 1460029 (9 pages)

(C) The Author

DOI: $10.1142 / \mathrm{S} 2010194514600295$

\title{
QUARK ORBITAL ANGULAR MOMENTUM AND FINAL STATE INTERACTIONS*
}

\author{
MATTHIAS BURKARDT \\ Department of Physics, New Mexico State University, Las Cruces, NM, USA \\ burkardt@nmsu.edu
}

Published 2 January 2014

\begin{abstract}
Definitions of orbital angular momentum based on Wigner distributions are used to discuss the connection between the Ji definition of the quark orbital angular momentum and that of Jaffe and Manohar. The difference between these two definitions can be interpreted as the change in the quark orbital angular momentum as it leaves the target in a DIS experiment. The mechanism responsible for that change is similar to the mechanism that causes transverse single-spin asymmetries in semi-inclusive deep-inelastic scattering.

Keywords: GPDs; TMDs; orbital angular momentum.
\end{abstract}

\section{Introduction}

Generalized Parton Distributions (GPDs) have been identified as a powerful tool to analyze the angular momentum decomposition of the nucleon. ${ }^{1}$ Furthermore GPDs can also be used to create truly three-dimensional images of the nucleon in the form of impact parameter dependent parton distributions. ${ }^{2}$ These images in a space where one dimension describes the light-cone momentum fraction and the other two dimensions describe the transverse position of the parton (relative to the transverse center of momentum) are complemented by Transverse Momentum dependent parton Distributions (TMDs). ${ }^{3}$ Wigner distributions provide a framework that allows a simultaneous description of GPDs and TMDs. ${ }^{4}$

Orbital Angular Momentum (OAM) correlates the position and momentum of partons. One can thus utilize Wigner distributions, which simultaneously embody the distribution of position and momentum, to define OAM. ${ }^{5,6}$ However, in the definition of these distributions, care must be applied to ensure gauge invariance. This can be accomplished by connecting any nonlocal correlation function with a Wilson-line gauge link, which requires specifying a path along which the vector

*This is an Open Access article published by World Scientific Publishing Company. It is distributed under the terms of the Creative Commons Attribution 3.0 (CC-BY) License. Further distribution of this work is permitted, provided the original work is properly cited. 
potential is evaluated. The choice of path raises the immediate issue of how the quantities defined using Wigner distributions (TMDs, OAM, ...) depend on that choice. The importance of this issue had become evident in the context of SingleSpin Asymmetries (SSAs). ${ }^{7}$ Indeed, while a straight-line gauge link definition of TMDs yields a vanishing Sivers effect, ${ }^{8,9}$ the correct gauge link relevant for TMDs in Semi-Inclusive Deep-Inelastic Scattering (SIDIS) involves a detour to light-cone infinity ${ }^{10}$ in order to properly include final-state interactions. In light-cone gauge, this subtlety had first been overlooked since in that gauge the Sivers effect solely arises from the contribution from the gauge-link piece at light-cone infinity. ${ }^{10}$

With Wigner distributions and OAM defined through them these issues arise all over again. ${ }^{6,11,12}$ The main goal of this note is to address that dependence of OAM defined through Wigner distributions on the choice of path for the gauge link and to interpret the resulting difference between common definitions of OAM.

\section{Angular Momentum Decompositions}

Since the famous EMC experiments revealed that only a small fraction of the nucleon spin is due to quark spins, ${ }^{13}$ there has been a great interest in 'solving the spin puzzle', i.e. in decomposing the nucleon spin into contributions from quark/gluon spin and orbital degrees of freedom. In this effort, the Ji decomposition ${ }^{1}$

$$
\frac{1}{2}=\frac{1}{2} \sum_{q} \Delta q+\sum_{q} L_{q}^{z}+J_{g}^{z}
$$

appears to be very useful: through GPDs, not only the quark spin contributions $\Delta q$ but also the quark total angular momenta $J_{q} \equiv \frac{1}{2} \Delta q+L_{q}^{z}$ (and by subtracting the spin piece also the the quark orbital angular momenta $L_{q}^{z}$ ) entering this decomposition can be accessed experimentally. In the Ji decomposition (1) the quark OAM is defined as the expectation value

$$
L_{q}^{z}=\int d^{3} r\left\langle P S\left|q^{\dagger}\left(\vec{r} \times \frac{1}{i} \vec{D}\right)^{z} q\right| P S\right\rangle /\langle P S \mid P S\rangle
$$

in a nucleon state polarized in the $+\hat{z}$ direction. Here $\vec{D}=\vec{\partial}-i g \vec{A}$ is the gaugecovariant derivative. The main advantages of this decomposition are that each term can be expressed as the expectation value of a manifestly gauge invariant local operator and that the quark total angular momentum $J^{q}=\frac{1}{2} \Delta q+L^{q}$ can be related to $\mathrm{GPDs}^{1}$ and is thus accessible in deeply virtual Compton scattering and deeply virtual meson production and can also be calculated in lattice gauge theory. Recent lattice calculations of $\mathrm{GPDs}^{14}$ yielded the surprising result that the light quark orbital angular momentum (OAM) is consistent with $L^{u} \approx-L^{d}$, i.e. $L^{u}+L^{d} \approx 0$. Unless there is a large contribution from disconnected quark loops, that had been so far omitteed, this would imply that $J^{g} \approx \frac{1}{2} \cdot 0.7$ represents the largest piece in the nucleon spin decomposition.

Jaffe and Manohar have proposed an alternative decomposition of the nucleon spin, which does have a partonic interpretation, ${ }^{15}$ and in which also two terms, $\frac{1}{2} \Delta q$ 
and $\Delta G$, are experimentally accessible

$$
\frac{1}{2}=\frac{1}{2} \sum_{q} \Delta q+\sum_{q} \mathcal{L}^{q}+\Delta G+\mathcal{L}^{g} .
$$

In this decomposition the quark OAM is defined as

$$
\mathcal{L}^{q} \equiv \int d^{3} r\left\langle P S\left|q_{+}^{\dagger}\left(\vec{r} \times \frac{1}{i} \vec{\partial}\right)^{z} q_{+}\right| P S\right\rangle /\langle P S \mid P S\rangle
$$

for a nucleon polarized in the $+\hat{z}$ direction. Here $q_{+} \equiv \frac{1}{2} \gamma^{-} \gamma^{+} q$ is the dynamical component of the quark field operators, and light-cone gauge $A^{+} \equiv A^{0}+A^{z}=0$ is implied. The residual gauge invariance can be fixed by imposing anti-periodic boundary conditions $\vec{A}_{\perp}\left(\mathbf{x}_{\perp}, \infty\right)=-\vec{A}_{\perp}\left(\mathbf{x}_{\perp},-\infty\right)$ on the transverse components of the vector potential. $\mathcal{L}$ also arises naturally in a light-cone wave function description of hadron states, where $\frac{1}{2}=\frac{1}{2} \sum_{q} \Delta q+\Delta G+\mathcal{L}$, in the sense of an eigenvalue equation, is manifestly satisfied for each Fock component individually. ${ }^{16}$

A variation of (1) has been suggested, ${ }^{17}$ where part of $L_{q}^{z}$ is interpreted as 'potential angular momentum'. As we will discuss in the following, the potential angular momentum also has a more physical interpretation as the effect from final state interactions. Other decompositions, in which only one term is experimentally accessible, will not be discussed in this brief note.

\section{TMDs and OAM from Wigner Distributions}

Wigner distributions can be defined as defined as off forward matrix elements of non-local correlation functions ${ }^{4,6,19}$

$$
\begin{aligned}
& W^{\mathcal{U}}\left(k^{+}=x P^{+}, \vec{b}_{\perp}, \vec{k}_{\perp}\right) \\
& \quad \equiv \int \frac{d^{2} \vec{q}_{\perp}}{(2 \pi)^{2}} \int \frac{d^{2} \xi_{\perp} d \xi^{-}}{(2 \pi)^{3}} e^{-i \vec{q}_{\perp} \cdot \vec{b}_{\perp}} e^{i\left(x P^{+} \xi^{-}-\vec{k}_{\perp} \cdot \vec{\xi}_{\perp}\right)}\left\langle P^{\prime} S^{\prime}\left|\bar{q}(0) \Gamma \mathcal{U}_{0 \xi} q(\xi)\right| P S\right\rangle
\end{aligned}
$$

with $P^{+}=P^{+\prime}, P_{\perp}=-P_{\perp}^{\prime}=\frac{q_{\perp}}{2}$. Throughout this paper, we will chose $\vec{S}=\vec{S}^{\prime}=\hat{\vec{z}}$. Furthermore, we will focus on the 'good' component by selecting $\Gamma=\gamma^{+}$. To ensure manifest gauge invariance, a Wilson line gauge link $\mathcal{U}_{0 \xi}$ connecting the quark field operators at position 0 and $\xi$ is included. The issue of choice of path for the Wilson line will be addressed below.

In terms of Wigner distributions, quark transverse momentum and OAM can be defined respectively as ${ }^{5}$

$$
\begin{aligned}
\left\langle\vec{k}_{\perp}\right\rangle_{\mathcal{U}} & =\int d x d^{2} \vec{b}_{\perp} d^{2} \vec{k}_{\perp} \vec{k}_{\perp} W^{\mathcal{U}}\left(x, \vec{b}_{\perp}, \vec{k}_{\perp}\right) \\
L_{\mathcal{U}} & =\int d x d^{2} \vec{b}_{\perp} d^{2} \vec{k}_{\perp}\left(\vec{b}_{\perp} \times \vec{k}_{\perp}\right)^{z} W^{\mathcal{U}}\left(x, \vec{b}_{\perp}, \vec{k}_{\perp}\right) .
\end{aligned}
$$

No issues with the Heisenberg uncertainty principle arise here since only perpendicular combinations of position $\vec{b}_{\perp}$ and momentum $\vec{k}_{\perp}$ are needed simultaneously in order to evaluate the integral for $L_{\mathcal{U}}$. 

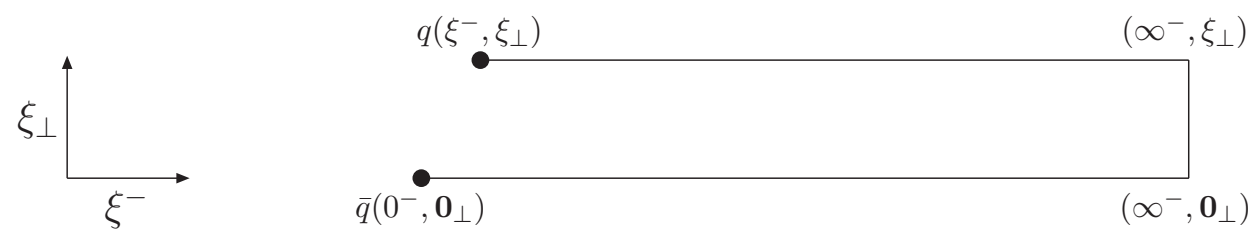

Fig. 1. Illustration of the path for the Wilson line gauge link $\mathcal{U}_{0 \xi}^{+L C}$ entering $W^{+L C}(5)$.

A straight line connecting 0 and $\xi$ for the Wilson line in $\mathcal{U}_{0 \xi}$ results in

$\left\langle\vec{k}_{\perp}^{q}\right\rangle_{\text {straight }} \equiv \int d x d^{2} \vec{b}_{\perp} d^{2} \vec{k}_{\perp} \vec{k}_{\perp} W^{\text {straight }}\left(x, \vec{b}_{\perp}, \vec{k}_{\perp}\right)=\frac{\int d^{3} \vec{r}\left\langle P S\left|\bar{q}(\vec{r}) \gamma^{+} \frac{1}{i} \vec{D} q(\vec{r})\right| P S\right\rangle}{\langle P S \mid P S\rangle}$,

which vanishes by PT invariance. ${ }^{9}$

However, depending on the context, other choices for the path in the Wilson link $\mathcal{U}$ should be made. Indeed, in the context of TMDs probed in SIDIS the path should be taken to be a straight line to $x^{-}=\infty$ along (or, for regularization purposes, very close to) the light-cone. This particular choice ensures proper inclusion of the Final State Interactions (FSI) experienced by the struck quark as it leaves the nucleon along a nearly light-like trajectory in the Bjorken limit. However, a Wilson line to $\xi^{-}=\infty$, for fixed $\vec{\xi}_{\perp}$ is not yet sufficient to render Wigner distributions manifestly gauge invariant, but a link at $\xi^{-}=\infty$ must be included to ensure manifest gauge invariance. While the latter may be unimportant in some gauges, it is crucial in light-cone gauge for the description of TMDs relevant for SIDIS. ${ }^{10}$

Let $\mathcal{U}_{0 \xi}^{+L C}$ be the Wilson path ordered exponential obtained by first taking a Wilson line from $\left(0^{-}, \overrightarrow{0}_{\perp}\right)$ to $\left(\infty, \overrightarrow{0}_{\perp}\right)$, then to $\left(\infty, \vec{\xi}_{\perp}\right)$, and then to $\left(\xi^{-}, \vec{\xi}_{\perp}\right)$, with each segment being a straight line (Fig. 1) [11]. The shape of the segment at $\infty$ is irrelevant as the gauge field is pure gauge there, but it is still necessary to include a connection at $\infty$ and for simplicity we pick a straight line. Likewise, with a similar 'staple' to $-\infty$ we define the Wilson path ordered exponential $\mathcal{U}_{0 \xi}^{-L C}$, and using those light-like gauge links we define

$$
\begin{aligned}
& W^{ \pm L C}\left(k^{+}=x P^{+}, \vec{b}_{\perp}, \vec{k}_{\perp}\right) \\
& \quad \equiv \int \frac{d^{2} \vec{q}_{\perp}}{(2 \pi)^{2}} \int \frac{d^{2} \xi_{\perp} d \xi^{-}}{(2 \pi)^{3}} e^{-i \vec{q}_{\perp} \cdot \vec{b}_{\perp}} e^{i\left(x P^{+} \xi^{-}-\vec{k}_{\perp} \cdot \vec{\xi}_{\perp}\right)}\left\langle P^{\prime} S^{\prime}\left|\bar{q}(0) \Gamma \mathcal{U}_{0 \xi}^{ \pm L C} q(\xi)\right| P S\right\rangle .
\end{aligned}
$$

This definition for $W^{+L C}$ the same as that in Ref.[11] and similar to that of $W_{L C}$ in Ref.[6], except that the link segment at $x^{-}=\infty$ was not included in the definition of $W_{L C}$ [6]. The Wilson like gauge link used to guarantee manifest gauge invariance is defined using a light-like 'staple, i.e. it is constructed using three straight line gauge links ${ }^{\mathrm{a}} \mathcal{U}_{0 \xi}^{+L C}=W_{0^{-} 0_{\perp}, \infty 0_{\perp}} W_{\infty 0_{\perp}, \infty \xi_{\perp}} W_{\infty \xi_{\perp}, \xi^{-} \xi_{\perp}}$ and similarly for $\mathcal{U}_{0 \xi}^{-L C}$.

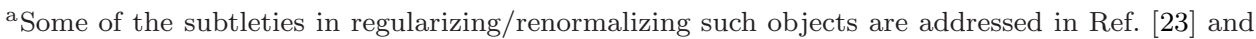
references therein.
} 
For 'advanced' boundary conditions, $\vec{A}_{\perp}\left(+\infty, \vec{r}_{\perp}\right)=0$ but $\vec{A}_{\perp}\left(-\infty, \vec{r}_{\perp}\right) \neq 0$, $W_{L C}$ from Ref. [6] becomes equal to $W^{+L C}$.

In light-cone gauge $A^{+}=0$ the Wilson lines to $x^{-}= \pm \infty$ become trivial and only the piece at $x^{-}=\infty$ remains. Although the gauge field at light-cone infinity $\vec{A}_{\perp}\left( \pm \infty, \vec{r}_{\perp}\right)$ cannot be neglected or set equal to zero in light-cone gauge, it can be chosen to satisfy anti-symmetric boundary conditions

$$
\vec{\alpha}_{\perp}\left(\vec{r}_{\perp}\right) \equiv \vec{A}_{\perp}\left(+\infty, \vec{r}_{\perp}\right)=-\vec{A}_{\perp}\left(-\infty, \vec{r}_{\perp}\right) .
$$

This choice maintains manifest PT (sometimes called 'light-cone parity') invariance.

Using $W^{ \pm L C}$, one can now introduce the corresponding average $\vec{k}_{\perp}^{q}$ as

$$
\begin{aligned}
\left\langle\vec{k}_{\perp}^{q}\right\rangle_{ \pm} & \equiv \int d x d^{2} \vec{b}_{\perp} d^{2} \vec{k}_{\perp} \vec{k}_{\perp} W^{ \pm L C}\left(x, \vec{b}_{\perp}, \vec{k}_{\perp}\right) \\
& =\frac{\int d^{3} \vec{r}\left\langle P S\left|\bar{q}(\vec{r}) \gamma^{+}\left(\frac{1}{i} \vec{\partial} \mp g \vec{\alpha}_{\perp}\left(\vec{r}_{\perp}\right)\right) q(\vec{r})\right| P S\right\rangle}{\langle P S \mid P S\rangle} .
\end{aligned}
$$

Eq. (10) differs from (7) by the matrix element of (in $A^{+}=0$ gauge)

$$
\begin{aligned}
\bar{q}(\vec{r}) \gamma^{+}\left[g A_{\perp}^{i}\left(\vec{r}_{\perp}\right)-g \alpha_{\perp}^{i}(\vec{r})\right] q(\vec{r}) & =-\bar{q}(\vec{r}) \gamma^{+} \int_{r^{-}}^{\infty} d z^{-} g \partial_{-} A_{\perp}^{i}\left(z^{-}, \vec{r}_{\perp}\right) q(\vec{r}) \\
& =-\bar{q}(\vec{r}) \gamma^{+} \int_{r^{-}}^{\infty} d z^{-} g G^{+i}\left(z^{-}, \vec{r}_{\perp}\right) q(\vec{r}),
\end{aligned}
$$

where $G^{+\perp}=\partial_{-} A^{\perp}$ is the gluon field strength tensor in $A^{+}=0$ gauge. Note that

$$
-\sqrt{2} g G^{+y} \equiv-g G^{0 y}-g G^{z y}=g\left(E^{y}-B^{x}\right)=g(\vec{E}+\vec{v} \times \vec{B})^{y}
$$

yields the $\hat{y}$ component of the color Lorentz force acting on a particle that moves with the velocity of light in the $-\hat{z}$ direction - which is the direction of the momentum transfer in DIS. Furthermore, the integration of the matrix element of (11) along the light-like trajectory of the ejected quark yields the average change in momentum: $\left\langle\vec{k}_{\perp}^{q}\right\rangle_{\text {straight }}=0$ while $\left\langle\vec{k}_{q}^{\perp}\right\rangle_{+L C}$ is the $\perp$ momentum relevant for SIDIS experiments. These observations motivate the semi-classical interpretation of the matrix element of (11) as the average transverse momentum of the ejected quark arising from the color-Lorentz force from the spectators as it leaves the target. ${ }^{22,21}$

In a general gauge, there is an additional contribution from the transverse derivative acting on the gauge links to/from $x^{-}=\infty$ and for example in an Abelian theory $\vec{\alpha}_{\perp}\left(\vec{r}_{\perp}\right)$ in (10) gets replaced by

$$
\alpha_{\perp}^{i}\left(\vec{r}_{\perp}\right) \rightarrow \alpha_{\perp}^{i}\left(\vec{r}_{\perp}\right)-\int_{r^{-}}^{\infty} d z^{-} \partial^{i} A^{+}\left(z^{-}, \vec{r}_{\perp}\right)=A_{\perp}^{i}\left(r^{-}, \vec{r}_{\perp}\right)-\int_{r^{-}}^{\infty} d z^{-} G^{+i}\left(z^{-}, \vec{r}_{\perp}\right),
$$

where $G^{+i}\left(z^{-}, \vec{r}_{\perp}\right)=\partial_{-} A^{i}-\partial^{i} A^{+}$. In the nonabelian case an additional commutator as well additional gauge links connecting the quark and the gluon operators arise. $^{24}$ Eq. (13) illustrates that the interpretation of the difference between the transverse momentum using light-cone staples and that using straight-line gauge links as the average color Lorentz force is gauge invariant. 
The same Wigner distributions that we used to define average transverse momentum can also be used to define OAM, yielding 6

$$
\begin{aligned}
L_{\text {straight }}^{q} & \equiv \int d x d^{2} \vec{b}_{\perp} d^{2} \vec{k}_{\perp}\left(\vec{b}_{\perp} \times \vec{k}_{\perp}\right)^{z} W^{\text {straight }}\left(x, \vec{b}_{\perp}, \vec{k}_{\perp}\right) \\
& =\frac{\int d^{3} \vec{r}\left\langle P S\left|q^{\dagger}(\vec{r})\left(\vec{r} \times \frac{1}{i} \vec{D}\right) q(\vec{r})^{z}\right| P S\right\rangle}{\langle P S \mid P S\rangle}=L_{J i}^{q},
\end{aligned}
$$

which is identical to the angular momentum that appears in the Ji-decomposition of the angular momentum for a nucleon (1), as well as $\left(A^{+}=0\right.$ gauge)

$$
\begin{aligned}
\mathcal{L}_{ \pm}^{q} & \equiv \int d x d^{2} \vec{b}_{\perp} d^{2} \vec{k}_{\perp}\left(\vec{b}_{\perp} \times \vec{k}_{\perp}\right)^{z} W^{ \pm L C}\left(x, \vec{b}_{\perp}, \vec{k}_{\perp}\right) \\
& =\frac{\int d^{3} \vec{r}\langle P S| \bar{q}(\vec{r}) \gamma^{+}\left[\vec{r} \times\left(\frac{1}{i} \vec{\partial} \mp g \vec{\alpha}_{\perp}\left(\vec{r}_{\perp}\right)\right]^{z} q(\vec{r})|P S\rangle\right.}{\langle P S \mid P S\rangle}
\end{aligned}
$$

and similar for the glue. $\mathcal{L}_{ \pm}^{q}$ is identical to the $\mathcal{L}^{q}$ appearing in the Jaffe-Manohar decomposition of the nucleon spin (3) as we will discuss below.

\section{Connections Between Different Definitions for OAM}

PT invariance implies $\mathcal{L}_{+}^{q}=\mathcal{L}_{-}^{q}[11]$. As a corollary, with anti-periodic boundary conditions (9) [12] the piece at $\pm \infty$ cancels in the average and both must thus be identical to the OAM appearing in the Jaffe-Manohar decomposition

$$
\mathcal{L}^{q}=\frac{1}{2}\left(\mathcal{L}_{+}^{q}+\mathcal{L}_{-}^{q}\right)=\mathcal{L}_{+}^{q}=\mathcal{L}_{-}^{q} .
$$

Therefore, although the gauge link at $x^{-}= \pm \infty$ is essential for the description of TMDs in $A^{+}=0$ gauge, ${ }^{10}$ it does not contribute to the OAM. .

To establish the connection with the orbital angular momentum entering the Ji-decomposition, we consider (for simplicity in light-cone gauge)

$$
\mathcal{L}^{q}-L^{q}=\mathcal{L}_{+}^{q}-L^{q}=\frac{\int d^{3} \vec{r}\left\langle P S\left|\bar{q}(\vec{r}) \gamma^{+}\left[\vec{r}_{\perp} \times\left(g \vec{A}_{\perp}(\vec{r})-g \vec{\alpha}_{\perp}\left(\vec{r}_{\perp}\right)\right)\right]^{z} q(\vec{r})\right| P S\right\rangle}{\langle P S \mid P S\rangle} .
$$

Here we replaced $\gamma^{0} \rightarrow \gamma^{+}$for a nucleon at rest in the definition for $L^{q}$ [20].

Using $(11,13)$ and the semi-classical interpretation of $-g G^{+i}\left(r^{-}, \vec{r}_{\perp}\right)$ as the $\perp$ force acting on the active quark along its trajectory we thus conclude that

$$
T^{z}\left(r^{-}, \vec{r}_{\perp}\right) \equiv-g\left(x G^{+y}\left(r^{-}, \vec{r}_{\perp}\right)-y G^{+x}\left(r^{-}, \vec{r}_{\perp}\right)\right)
$$

represents the $\hat{z}$ component of the torque that acts on a particle moving with (nearly) the velocity of light in the $-\hat{z}$ direction - the direction in which the ejected quark moves. Thus the difference between the (forward) light-cone definition $L^{+L C}=L_{J M}$ and the local definition $L_{\text {straight }}=L_{J i}$ of the orbital angular 


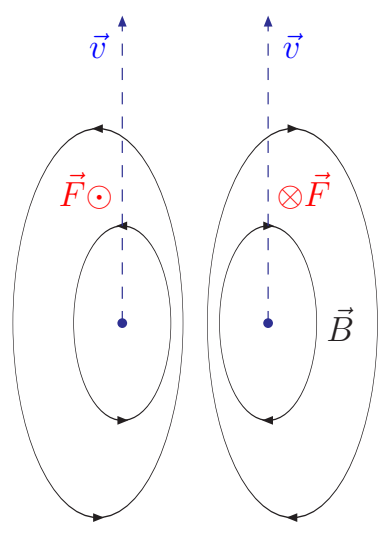

a.)

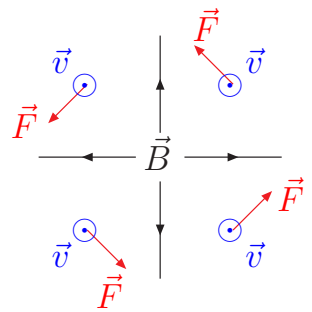

b.)

Fig. 2. Illustration of the torque acting on the struck quark in the $-\hat{z}$ direction through a colormagnetic dipole field caused by the spectators. a.) side view; b.) top view. In this example the $\hat{z}$ component of the torque is negative as the quark leaves the nucleon.

momentum is the change in orbital angular momentum as the quark moves through the color field created by the spectators

$$
\mathcal{L}^{q}-L^{q}=\frac{\int d^{3} \vec{r}\left\langle P S\left|\bar{q}(\vec{r}) \gamma^{+} \int_{r^{-}}^{\infty} d z^{-} T^{z}\left(z^{-}, \vec{r}_{\perp}\right) q(\vec{r})\right| P S\right\rangle}{\langle P S \mid P S\rangle} .
$$

Therefore, while $L^{q}$ represents the local and manifestly gauge invariant OAM of the quark before it has been struck by the $\gamma^{*}, \mathcal{L}^{q}$ represents the gauge invariant OAM after it has left the nucleon and moved to $r^{-}=\infty$. This physical interpretation of the difference between the Jaffe-Manohar definition of quark OAM with a light-cone staple and the local definition ( Ji) represents the main result of this paper.

It is easy to see that a torque as appearing in (19) may exist by considering the example of a quark moving through a (color-) magnetic dipole field caused by the spectators. Because of the overall color-neutrality, this is similar to a positively charged particle moving through the magnetic field caused by negative spectators in QED. For spectator spins/OAMs that are oriented in the $+\hat{z}$ axis one would thus expect a dipole field as shown in Fig. 2. All quarks ejected in the $-\hat{z}$ direction pass through the region of outward pointing radial magnetic field component, but only those originating in the bottom portion also move through regions of inward pointing radial component, i.e. for quarks ejected in the $-\hat{z}$ direction the regions of outward pointing radial component dominate. One would thus expect more torque in the $-\hat{z}$ direction than in $+\hat{z}$ direction. This example not only illustrates that the net change in OAM as the quark leaves the nucleon is nonzero, but also suggests 
what the sign of $\mathcal{L}^{q}-L^{q}$ might be: for $d$ quarks the spins of the spectators are positively correlated with the nucleon spin, corresponding to a situation similar to the one depicted in Fig. 2, and $\mathcal{L}^{q}-L^{q}$ should thus be negative. For $u$ quarks the situation is less obvious since there should be a partial cancellation between the $d$ quark spectator and the $u$ quark spectator. For an positron (electron) moving through its own dipole field in QED the magnetic dipole field is reversed. This illustrates why $\mathcal{L}^{e}-L^{e}$ is positive for an electron. ${ }^{20}$

\section{Summary}

The OAM appearing in the Jaffe-Manohar formalism are identical to Wigner function based definitions of OAM utilizing light-cone staples. We have used this result to understand the difference between the Jaffe-Manohar definition of OAM and Ji's local manifestly gauge invariant definition of OAM can be related to the torque that acts on a quark in longitudinally polarized DIS. In other words., while one definition $(\mathrm{Ji})$ yields the net OAM quarks before absorbing the virtual photon, the (light-cone staple) Wigner distribution based definition (JM) yields the net OAM after the quark has escaped to infinity. We thus now understand the physics through which these two definitions are related to one another.

This is very similar to the situation in the context of TMDs where the difference between the average quark transverse momentum after it has left the target (from Sivers function) and before it has left the target (where it is zero), can be related to the difference of TMDs defined with a light-cone staple shaped Wilson line gauge link versus one defined with a straight-line gauge link.

Unfortunately, no experiment has been identified to measure the OAM of quarks after they have been ejected in DIS. Nevertheless, we believe that the above interpretation will help to develop a more complete picture of the nucleon spin.

\section{Acknowledgments:}

I would like to thank G. Schnell for stimulating discussions during early stages of this work. This work was partially supported by the DOE (DE-FG03-95ER40965).

\section{Appendix A. Classical Motion in a Magnetic Dipole Field}

A magnetic dipole polarized in the $\hat{\vec{z}}$ direction $\vec{\mu}=\mu \hat{\vec{z}}$ has a magnetic field

$$
\vec{B}=\vec{\nabla} \times \vec{A}=\frac{\mu_{0}}{4 \pi}\left[\frac{3 \vec{r}(\vec{\mu} \cdot \vec{r})}{r^{5}}-\frac{\vec{\mu}}{r^{3}}\right]
$$

where $\vec{A}=\frac{\mu_{0}}{4 \pi} \frac{\vec{\mu} \times \overrightarrow{\hat{r}}}{r^{2}}$ satisfies $A^{z}=0$. For particle with charge $q$ and $\vec{v}=-\hat{\vec{z}}$ that moves from $z=z_{0}$ to $z_{0}=-\infty$ this implies

$$
T^{z}=q \vec{r} \times(\vec{v} \times \vec{B})=\frac{-3 q \mu_{0} \mu z}{4 \pi r^{3}}\left[\frac{x^{2}+y^{2}}{r^{2}}\right] .
$$


Hence the change in OAM due to the FSI reads (note: $d t=-d z$ )

$$
\Delta L_{F S I}^{z}=\int_{0}^{\infty} d t T^{z}=-\int_{z_{0}}^{-\infty} d z T^{z}=\int_{-\infty}^{z_{0}} d z T^{z}=\frac{q \mu_{0} \mu}{4 \pi} \frac{x^{2}+y^{2}}{\left(x^{2}+y^{2}+z_{0}^{2}\right)^{\frac{3}{2}}}
$$

which happens to be identical to $q[\vec{r} \times \vec{A}]^{z}$ at $\vec{r}=\left(x, y, z_{0}\right)$.

In this example, Ji's OAM should be identified with $L \equiv L_{J i}^{z}=\vec{r} \times(\vec{p}-q \vec{A})$, while $\mathcal{L} \equiv L_{J M}^{z}=\vec{r} \times \vec{p}$ (note that $\vec{A}=0$ at $z=\infty$ in this example). Therefore

$$
\mathcal{L}-L=L_{J M}^{z}-L_{J i}^{z}=q[\vec{r} \times \vec{A}]^{z}=\Delta L_{F S I}^{z}
$$

consistent with Eq. (19).

\section{References}

1. X. Ji, Phys. Rev. Lett. 78, 610 (1997).

2. M. Burkardt, Phys. Rev. D 62, 071503 (2000); 66, 119903(E) (2002).

3. D.Boer, P.J.Mulders, and F.Pijlman, Nucl. Phys. B 667, 201 (2003); see also R.D.Tangerman and P.J.Mulders, Phys. Rev. D 51, 3357 (1995).

4. A.V. Belitsky, X. Ji, and F. Yuan, Phys. Rev. D 69, 074014 (2004).

5. C. Lorcé and B. Pasquini, Phys. Rev. D 84, 014015 (2011); C. Lorce, B. Pasquini, X. Xiong, and F. Yuan, Phys. Rev. D 85, 114006 (2012).

6. X. Ji, X. Xiong, and F. Yuan, Phys. Rev. Lett. 109, 152005 (2012).

7. S.J. Brodsky, D.S. Hwang and I. Schmidt, Phys. Lett. B 530, 99 (2002).

8. D.W. Sivers, Phys. Rev. D 43, 261 (1991).

9. J.C. Collins, Phys. Lett. B 536, 43 (2002).

10. A.V. Belitsky, X. Ji, and F. Yuan, Nucl. Phys. B 656, 165 (2003); D.Boer, P.J.Mulders, and F.Pijlman, Nucl. Phys. B 667, 201 (2003).

11. Y. Hatta, Phys. Rev. D 84, 041701 (2011); Phys. Lett. B 708, 186 (2012).

12. C. Lorcé, Phys.Lett. B 719, 185 (2013).

13. J. Ashman et al (EMC), Phys. Lett. B 206, 364 (1988); Nucl. Phys. B 328, 1 (1989).

14. Ph. Hägler et al. (LHPC Collaboration), Phys. Rev. D 77, 094502 (2008).

15. R.L. Jaffe and A. Manohar, Nucl. Phys. B 337, 509 (1990).

16. S.J. Brodsky et al., Nucl. Phys. B 593, 311 (2001).

17. M. Wakamatsu, Phys. Rev. D 81, 114010 (2010); Eur. J. Phys. A 44, 297 (2010); Phys. Rev. D 85, 114039 (2012).

18. M. Burkardt, Phys. Rev. D 69, 091501 (2004).

19. S. Meissner, A. Metz, and M. Schlegel, J. High Energy Phys. 08, 056 (2009).

20. M. Burkardt and H. BC, Phys. Rev. D 79, 071501 (2009).

21. M. Burkardt, arXiv:08103589 (2008).

22. J. Qiu and G. Sterman, Phys. Rev. Lett. 67, 2264 (1991).

23. J.C. Collins, Acta Phys. Polon. B 34, 3103 (2003); S.M. Aybat et al., Phys. Rev. D 85, 034043 (2012); I. Cherednikov, Phys. Part. Nucl. 44, 250 (2013).

24. M. Burkardt, arXiv:1205.2916 (2012). 